\title{
Submitted: Diagnostic accuracy of ultrasonography in adults Accepted: with obstructive jaundice
} 23.04.2020

Published: 15.06.2020

\section{Keywords}

ultrasonography, sensitivity, accuracy, biliary ducts, obstructive jaundice

\author{
Olufunke O. Fadahunsi ${ }^{1}$, Bolanle O. Ibitoye ${ }^{1}$, Adewale O. Adisa², \\ Olusegun I. Alatise², Victor A. Adetiloye', Bukunmi Michael Idowu ${ }^{3}$ \\ ${ }^{1}$ Department of Radiology' Obafemi Awolowo University Teaching Hospitals Complex, Ile-Ife, \\ Osun State, Nigeria \\ ${ }^{2}$ Department of Surgery², Obafemi Awolowo University Teaching Hospitals Complex, Ile-Ife, \\ Osun State, Nigeria \\ ${ }^{3}$ Department of Radiology, Union Diagnostics and Clinic Services Plc, Yaba, Lagos, Nigeria \\ Correspondence: Dr. Bukunmi M. IDOWU, Department of Radiology, Union Diagnostics \\ and Clinic Services Plc, Yaba, Lagos, Nigeria; e-mail:ibmcontacts@gmail.com
}

DOI: $10.15557 / J o U .2020 .0016$

\begin{abstract}
Aim of the study: To determine the sensitivity and specificity of ultrasound for detecting the causes of obstructive jaundice. Materials and methods: Eighty adult patients with clinical and biochemical features of obstructive jaundice were enrolled in this study. The causes, degrees and levels of ductal obstruction were evaluated sonographically via the transabdominal route. The ultrasonographic diagnoses were correlated with surgical findings and histopathological diagnoses. Results: The age range was 16 to 82 years, with a mean of 51.06 \pm 14.95 years. The peak age group was the sixth decade with $23(28.8 \%)$ patients. There were nearly twice as many females as males, with $28(35 \%)$ males and $52(65 \%)$ females, giving a male to female ratio of $1: 1.9$. On ultrasound, pancreatic carcinoma $(28.0 \%)$ and choledocholithiasis $(21.3 \%)$ were the most common malignant and benign causes of obstructive jaundice, respectively. Hepatocellular carcinoma (1.3\%) was the least common etiology. There was a strong correlation between the definitive diagnosis and the sonographic level of obstruction. The overall sensitivity of ultrasound for detecting the cause of obstruction was $76.6 \%$, while the specificity was $98 \%$. Conclusion: Ultrasonography is a reliable imaging modality for diagnosing the cause and level of obstruction in surgical jaundice. The sensitivity is adequate to aid the early institution of surgical intervention, thereby preventing morbidity and mortality that may accompany late interventions in our setting.
\end{abstract}

\section{Introduction}

Obstructive jaundice, otherwise called surgical jaundice, is a common condition globally ${ }^{(1-3)}$. Common causes of obstruction of the extrahepatic biliary tree in adult patients include choledocholithiasis, chronic pancreatitis, as well as neoplasms of the pancreas, gallbladder, biliary tract, or the ampulla of Vater. Other less prevalent causes include metastasis to the porta hepatis, hepatic tumour adjacent to the hilum, perihepatic lymphadenopathy, sclerosing cholangitis and other forms of cholangitis ${ }^{(3,4)}$.
Many patients present with advanced surgical jaundice requiring various examinations to determine the cause and level of obstruction. The array of invasive and noninvasive radiological techniques commonly employed for investigating hepatobiliary lesions includes computed tomography (CT), percutaneous transhepatic cholangiography (PTC), endoscopic ultrasound (EUS), endoscopic retrograde cholangiopancreatography (ERCP), helical CT cholangiography, Magnetic Resonance Cholangiopancreatography (MRCP), radionuclide imaging, and ultrasonography ${ }^{(1,5-8)}$. These examinations are 
useful to varying degrees for assessing both the cause and site of obstruction. However, ultrasonography is the least invasive initial imaging modality for the evaluation of jaundiced patients ${ }^{(4-6,9,10)}$.

In resource-poor settings, ultrasound is often the only readily available method, with many of the other modalities being too expensive or unavailable to many patients. Furthermore, abdominal ultrasound is considered to be the first initial imaging procedure in obstructive jaundice ${ }^{(1,5,11,12)}$. Ultrasonography could also be used for some imaging-guided hepatobiliary interventions.

This study aims to determine the sensitivity and specificity of ultrasound in detecting the causes of obstructive jaundice in our locality, where more sophisticated imaging modalities may not be readily available on a routine basis.

\section{Methods}

This was a hospital-based, cross-sectional study of eighty consecutive adult patients referred for abdominal ultrasound in the Radiology Department of our hospital on account of clinical and biochemical features of obstructive jaundice. The study was approved by the Ethics and Research Committee of the institution (Approval number: IRB/IEC/0004553), and was conducted in accordance with the Declaration of Helsinki (2013).

This multidisciplinary study involved the departments of Radiology, General Surgery and Histopathology. All patients were referred to the Radiology Department from the Surgery Department for sonographic evaluation. They were then referred back to the Surgery Department, where they had surgery. Later, histopathological examinations of surgical biopsy specimens were performed where applicable. The surgical and/or histopathology reports were ultimately correlated with the ultrasonographic diagnosis.

All adult patients with clinical and biochemical (liver function test) features of obstructive jaundice were enrolled consecutively. Written informed consent was obtained from all participants after the study had been adequately explained to them. The following patients were excluded from the study: children with jaundice ( $<16$ years old) and adult patients with features of pre-surgical jaundice or in whom liver function test (LFT) did not show features of obstruction. All the patients underwent sonographic examinations before the treatment/intervention was instituted. They were all scanned by the first author who was a $5^{\text {th }}$ year radiology resident, under close supervision of two consultant radiologists with 17-28 years' experience.

A Mindray real-time ultrasound scanner model DC-6 (Shenzhen Mindray Bio-medical Electronics, Nanshan, Shenzhen, China) with Doppler facilities and a curvilinear transducer (frequency $=2.5-5.0 \mathrm{MHz}$ ) was used.

The patients were examined after an overnight fast of 8 hours, with the stomach distended with water to remove excess gas, and to create an acoustic window to view the pancreas adequately.

With the patient in the supine position, a coupling gel was applied to the exposed abdomen in the right upper quadrant. Scanning was done in both longitudinal and transverse planes. The patient was then placed in the left posterior oblique or left lateral decubitus position in order to scan the intra and extra-hepatic ductal systems. The common bile duct (CBD) was imaged by placing the transducer below the right costal margin in the region of the midclavicular line, with the patient in the oblique position. When visualized, the CBD was traced from the hepatic hilum to the retro-pancreatic portion and down to the papilla. It was imaged along its entire length, and its diameter was measured at three levels viz: at the porta hepatis, close to the liver, and distally near the head of the pancreas ${ }^{(13)}$. The diameters of the common hepatic duct and the common bile duct were measured, with $6 \mathrm{~mm}$ taken as the upper limit of normal for both. Above $6 \mathrm{~mm}$ was considered dilated $(1 \mathrm{~mm}$ per decade was added to this upper limit in patients who were older than 60 years). The intra-hepatic ducts were also evaluated. The hepatic hilum and gall bladder were assessed with the patient in the left lateral decubitus position. We used color flow Doppler to differentiate tumefactive sludge from the gallbladder mass. Doppler examination was also used to assess vascularization of the gallbladder wall and cancer or formation of a twinkling artifact in a choledocholithiasis.

Where relevant, scanning was performed with the subject sitting erect in order to assess whether gallstones were mobile. The gallbladder wall thickness was regarded as normal when it was $\leq 3 \mathrm{~mm}$, including the measurement of its anterior wall ${ }^{(13)}$.

The spleen, stomach, bowel loops, and para-aortic regions were also scanned to exclude pathologies. Ascites was noted, when present. Sonographic diagnosis of the wide variety of underlying etiologies was based on published, well-established criteria. Sonographic findings and diagnoses were finally compared to operative findings and/or histopathological reports.

The data were analysed using IBM SPSS Statistics software version 22.0 for windows (IBM Corp., Armonk, N.Y., USA). The test of normality was performed with the Kolmogorov Smirnov's test. Chi-square was used to compare categorical data, while analysis of variance (ANOVA) was used to compare continuous data between groups, with Scheffe/Bonferroni post-hoc analysis done for intergroup differences. Continuous variables were presented as mean \pm SD (standard deviation), while categorical variables were expressed as percentages and frequencies. Ultrasonographic diagnoses were compared to the final surgical and/or histopathologic diagnoses to calculate the sensitivity, specificity, positive predictive value, negative predictive value, and accuracy of ultrasound. Statistical significance was set at $p \leq 0.05$. 
Tab. 1. Age and sex of patients with obstructive jaundice

\begin{tabular}{|l|c|c|c|}
\hline \multirow{2}{*}{ Age (years) } & \multicolumn{3}{|c|}{ Sex } \\
\cline { 2 - 4 } & \multicolumn{3}{|c|}{ Frequency (\%) } \\
\cline { 2 - 4 } & Male & Female & Total \\
\hline $16-19$ & $2(2.50)$ & $1(1.25)$ & $3(3.75)$ \\
\hline $20-29$ & $2(2.50)$ & $5(6.25)$ & $7(8.75)$ \\
\hline $30-39$ & $1(1.25)$ & $6(7.50)$ & $7(8.75)$ \\
\hline $40-49$ & $4(5.00)$ & $8(10.00)$ & $12(15.00)$ \\
\hline $50-59$ & $8(10.00)$ & $15(18.75)$ & $23(28.75)$ \\
\hline $60-69$ & $11(13.75)$ & $11(13.75)$ & $22(27.50)$ \\
\hline $70-79$ & $0(0.00)$ & $4(5.00)$ & $4(5.00)$ \\
\hline $80-89$ & $0(0.00)$ & $2(2.50)$ & $2(2.50)$ \\
\hline Total & $\mathbf{2 8 ( 3 5 )}$ & $\mathbf{5 2 ( 6 5 )}$ & $\mathbf{8 0 ( 1 0 0 )}$ \\
\hline
\end{tabular}

Tab. 2. Ultrasound diagnosis of obstructive jaundice

\begin{tabular}{|l|c|c|}
\hline Ultrasound Diagnosis & $\begin{array}{c}\text { Number of } \\
\text { patients }\end{array}$ & Percentage (\%) \\
\hline Pancreatic carcinoma & 23 & 28.8 \\
\hline Choledocholithiasis & 15 & 18.7 \\
\hline Gallbladder carcinoma & 13 & 16.3 \\
\hline Periampullary carcinoma & 8 & 10.0 \\
\hline Cholangiocarcinoma & 4 & 5.0 \\
\hline Metastatic lesion & 4 & 5.0 \\
\hline Choledocholithiasis + Cirrhosis & 3 & 3.7 \\
\hline Cholelithiasis & 3 & 3.8 \\
\hline Cholecystitis & 2 & 2.5 \\
\hline Lymphoproliferative disease & 2 & 2.5 \\
\hline Gastric carcinoma & 1 & 1.2 \\
\hline Hepatocellular carcinoma & 1 & 1.3 \\
\hline Liver cirrhosis & 1 & 1.2 \\
\hline Total & $\mathbf{8 0}$ & $\mathbf{1 0 0}$ \\
\hline
\end{tabular}

\section{Results}

Eighty cases of obstructive jaundice were included in this study. The age range was 16 to 82 years, with a mean of $51.06 \pm 14.95$ years. The peak age group was the sixth decade with $23(28.8 \%)$ patients. There were nearly twice as many females as males, with 28 (35\%) males and $52(65 \%)$ females, giving a male to female ratio of 1:1.9. (Tab. 1).

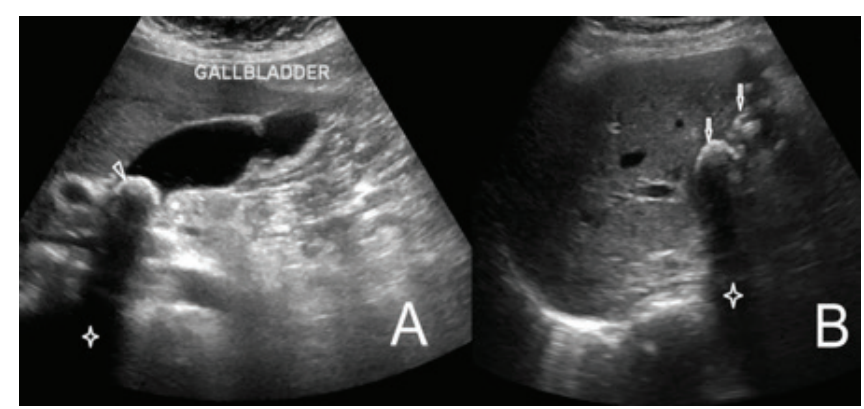

Fig. 1. Longitudinal sonogram of the gallbladder showing gallstone disease in 2 patients as $\mathbf{A}$. an impacted echogenic structure in the gallbladder neck (arrowhead) with acoustic shadowing (star), and B. multiple echogenic structures within a contracted gallbladder giving a wall-echo shadow complex (downward arrows)

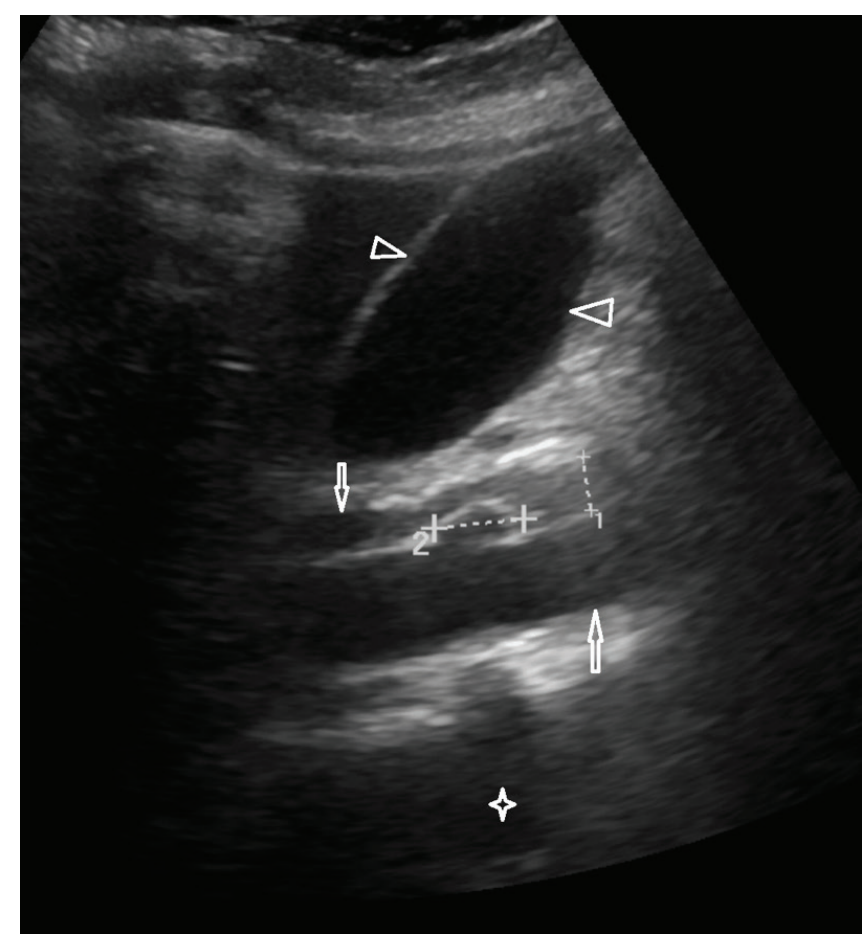

Fig. 2. Choledocholithiasis - longitudinal sonogram of the hepatic hilum showing a large calculus with posterior acoustic shadow (star) within dilated common bile duct (downward arrow); the gallbladder (arrowheads) and portal vein (upward arrow) are also shown

On ultrasound, pancreatic carcinoma 23 (28.8\%), choledocholithiasis 15 (18.7\%), and gallbladder carcinoma 13 $(16.3 \%)$ were the most prevalent diagnoses. Less frequent findings included periampullary carcinoma 8 (10.0\%), cholangiocarcinoma $4(5.0 \%)$, and metastatic lesions. Other findings are shown in Tab. 2 and Fig. 1, Fig. 2, Fig. 3.

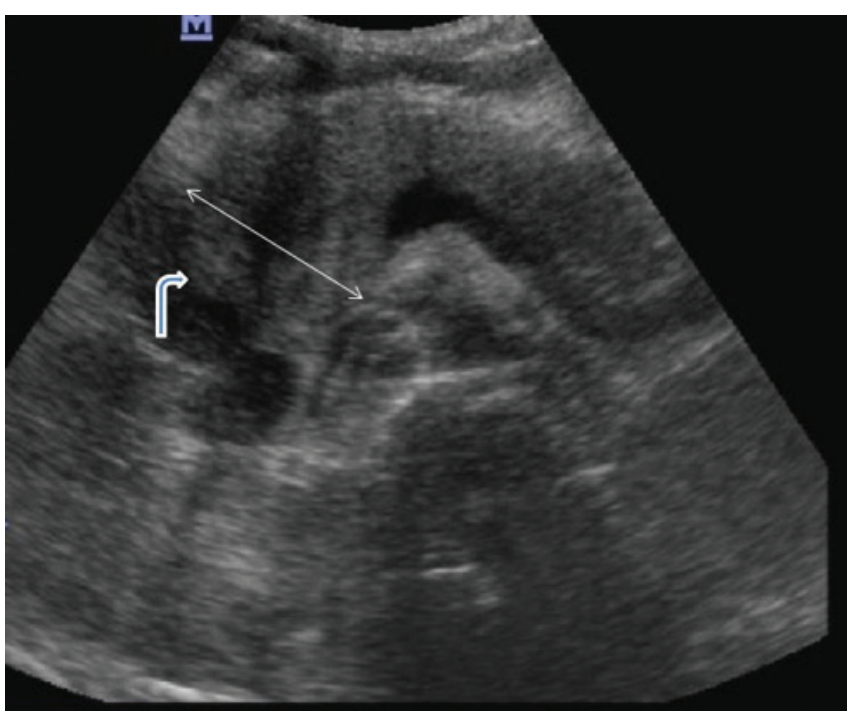

Fig. 3. Transverse sonogram of the pancreas showing a manifestation of pancreatic carcinoma as an enlarged, lobulated and hypoechoic pancreatic head (curved and double arrows) 
Tab. 3. Prevalence of dilatation of biliary ducts with level of obstruction

\begin{tabular}{|c|c|c|c|c|}
\hline \multicolumn{5}{|c|}{ a) Prevalence of dilatation of biliary ducts with mean diameters } \\
\hline \multirow{2}{*}{ Biliary duct } & \multirow{2}{*}{ Number of patients $\mathbf{N}(\%)$} & \multicolumn{3}{|c|}{ Diameter of the duct (mm) } \\
\hline & & Mean \pm SD & Median & Range \\
\hline CBD & $60(75.0)$ & $13.7 \pm 7.3$ & 13.9 & $3.0-32.0$ \\
\hline $\mathrm{CHD}$ & $15(18.7)$ & $12.8 \pm 4.8$ & 12.0 & $4.5-28.0$ \\
\hline No dilatation & $5(6.3)$ & - & - & - \\
\hline Total & $80(100.0)$ & - & - & - \\
\hline \multicolumn{5}{|c|}{ b) Comparison of degree of biliary dilatation with level of obstruction } \\
\hline Mean biliary dilatation & Intrapancreatic obstruction & $\begin{array}{c}\text { Suprapancreatic } \\
\text { obstruction }\end{array}$ & Hilar obstruction & $p$-value* \\
\hline CBD Diameter (Mean \pm SD mm) & $19.4 \pm 5.3$ & $9.8 \pm 4.2$ & $6.4 \pm 1.9$ & $<0.001$ \\
\hline CHD Diameter (Mean \pm SD mm) & $15.2 \pm 5.2$ & $11.6 \pm 2.9$ & $10.7 \pm 2.1$ & $<0.001$ \\
\hline
\end{tabular}

A total of 75 patients $(93.7 \%)$ had dilatation of the extrahepatic ducts. Forty $(50 \%)$ patients had dilatation of the extra-hepatic biliary tree up to the distal CBD, while dilatation up to the proximal CBD was seen in 20 (25\%) patients. Common hepatic ductal dilatation was seen in 15 patients $(18.8 \%)$. Only $5(6.3 \%)$ of the patients had no significant ductal dilatation on ultrasound. The CBD diameters ranged from $3.0 \mathrm{~mm}$ to $32.0 \mathrm{~mm}$, with a mean of $13.7 \pm 7.3 \mathrm{~mm}$, while the common hepatic duct diameters ranged from 4.5 $\mathrm{mm}$ to $28.0 \mathrm{~mm}$, with a mean of $12.8 \pm 4.8 \mathrm{~mm}$. When visualized, the pancreatic duct diameter ranged between $2.9 \mathrm{~mm}$ and $13.0 \mathrm{~mm}$, with a mean of $5.1 \pm 2.6 \mathrm{~mm}$ (Tab. 3A).

A comparison of the mean CBD diameters with respect to the level of obstruction using analysis of variance (ANOVA) revealed a statistically significant difference ( $p<0.001$; Tab. 3B). Posthoc tests (Bonferroni and Scheffe) showed that the statistically significant difference between the three groups was mainly due to a higher CBD diameter in the intra-pancreatic obstruction group than in the other two groups $(p<0.001)$. The difference in the mean CBD diameter between the hilar and supra-pancreatic obstruction groups was not statistically significant $(p=$ 0.12 ). A similar pattern was observed between the groups when the mean common hepatic duct diameters were compared.
The preoperative ultrasonographic diagnoses were correlated with the definitive diagnoses at surgery and histopathological reports of operative biopsies for malignant conditions (Tab. 4). The overall sensitivity of ultrasound in detecting the etiology of obstructive jaundice was $76.6 \%$, while the specificity was $98 \%$. For specific disease entities, the values of sensitivity and specificity were $81.0 \%$ and $94.6 \%$, respectively, for pancreatic carcinoma, $70.7 \%$ and $93.7 \%$ for choledocholithiasis, and $80 \%$ and $98 \%$ for gallbladder carcinoma. The other hepatobiliary pathologies are shown in Tab. 4.

\section{Discussion}

Intraoperative dissection of the hepatopancreatobiliary tree can be challenging for surgeons ${ }^{(1,3,14)}$, and precise preoperative diagnosis of the exact site of obstruction is beneficial for planning surgical interventions ${ }^{(3)}$. In resource-rich settings, this is usually accomplished by a combination of different imaging and endoscopic modalities, some of which may not be readily available for surgeons in resource-limited settings.

This study investigated the diagnostic accuracy of ultrasonography for reaching a definitive diagnosis in patients with

Tab. 4. Comparison of Ultrasound Diagnosis with Final Diagnosis (Surgical/Histological)

\begin{tabular}{|l|c|c|c|c|c|}
\hline Ultrasound diagnosis & Accuracy (\%) & PPV (\%) & NPV (\%) & Sensitivity (\%) & Specificity (\%) \\
\hline Pancreatic carcinoma & 90.9 & 85.0 & 92.0 & 81.0 & 94.6 \\
\hline Choledocholithiasis & 88.8 & 75.0 & 92.0 & 70.7 & 93.7 \\
\hline Liver cirrhosis & 94.9 & 50.0 & 97.0 & 50.0 & 97.0 \\
\hline Hepatocellular carcinoma & 100.0 & 100.0 & 100.0 & 100.0 & 100.0 \\
\hline Gastric carcinoma & 98.8 & 100.0 & 98.7 & 50.0 & 100.0 \\
\hline Gall bladder carcinoma & 94.0 & 92.0 & 95.0 & 80.0 & 98.0 \\
\hline Cholangitis & 97.5 & 0.0 & 97.5 & 100 & 100.0 \\
\hline Cholangiocarcinoma & 100.0 & 100.0 & 100.0 & 100.0 & 100.0 \\
\hline Periampullary carcinoma & 96.3 & 62.5 & 100.0 & 100.0 & 96.0 \\
\hline Lymphoproliferative disease & 98.8 & 50.0 & 100.0 & 100.0 & 90.0 \\
\hline Metastatic disease & 98.8 & 100.0 & 99.0 & & 100.0 \\
\hline PPV - positive predictive value; NPV - negative predictive value & & & \\
\hline
\end{tabular}


obstruction of the extra-hepatic biliary tree. We observed a good correlation between the definitive diagnosis and sonographic diagnosis, with an overall sensitivity of $76.6 \%$ and specificity of $98 \%$. These findings are similar to the study by Kumar et al. (15) $^{(1)}$ which concluded that ultrasonography as a single radiological investigation was sufficient in the evaluation of most patients with obstructive jaundice, with a sensitivity of $84 \%$ in detecting its cause. In contrast, Dewbury et al. ${ }^{(16)}$ obtained a lower sensitivity of ultrasound for detecting the cause of obstructive jaundice, with a value of $58 \%$. This disparity could be attributed to a higher resolution of the modern ultrasound system we used, and possibly a lower level of experience at the time their study was carried out in 1979.

The most frequent level of obstruction in our study was at the intra-pancreatic common bile duct, as seen in $40(50 \%)$ of the patients, followed by the supra-pancreatic common bile duct in $20(25 \%)$ patients. Fifteen $(18.8 \%)$ patients had obstruction at the level of the porta hepatis, while there was no sonographic evidence of obstruction in $5(6.3 \%)$ patients. Similar findings were noted by Upadhyaya et al. ${ }^{(17)}$ in India, who noted that $27 \%$ of their patients had obstruction at the level of the porta hepatis, $34 \%$ at the supra-pancreatic level, and $38 \%$ at the intra-pancreatic level. However, Ghimire et al. ${ }^{(18)}$ in Nepal reported that their patients had obstruction at the hilar level in $38 \%$ of cases, and supra-pancreatic duct obstruction in $33 \%$ of cases, while $28 \%$ of their patients had obstruction at the intra-pancreatic level. Differences in the etiology of the obstruction may account for this variation.

The mean diameters of the common bile duct (CBD) and common hepatic duct (CHD) in intra-pancreatic bile duct obstruction were statistically significantly higher than ductal diameters recorded in obstructions at other levels. This implies that a more distal obstruction of the biliary tree is associated with a significantly worse proximal ductal dilatation compared to other levels of obstruction. This may account for a relatively higher sensitivity of ultrasound in detecting lower duct obstruction in our study compared with that of Ghimire et al. ${ }^{(18)}$

Our sensitivity and specificity values in diagnosing pancreatic carcinoma on ultrasound were $81 \%$ and $94.6 \%$, respectively. This is quite remarkable, when compared with the findings reported by Admassie et al. ${ }^{(9)}$, who obtained sensitivity and specificity values of $50 \%$ and $90 \%$, respectively. The higher sensitivity noted in our study may be due to greater dilatation of the common bile and hepatic ducts (i.e. worse severity), higher resolution of the ultrasound equipment, and the technique of examining patients in the anterior oblique position with the stomach fully distended with water. D'Onofrio et al. ${ }^{(19)}$ reported a sensitivity of $88 \%$, a specificity of $97 \%$, and an accuracy of $96 \%$ in their study in which they employed contrast agents to enhance sonographic images.

The sensitivity, specificity, and accuracy of ultrasound in diagnosing gallbladder carcinoma in this study were $80 \%$, $90 \%$, and $94 \%$, respectively. This is lower than the $100 \%$ sensitivity level reported by Upadhyaya et al. ${ }^{(17)}$ We attribute this to an additional unrecognized sonographic feature of advanced gallbladder carcinoma that we encountered: nonvisualization of the gall bladder along with an echogenic structure casting posterior acoustic shadows adjacent to the porta hepatis. This was initially misdiagnosed as chronic cholecystitis with choledocholithiasis. At surgery, however, the findings were that of advanced infiltrating gallbladder carcinoma, walled off by bowel loops. This sonographic feature had been documented before in a report by Alatise et al. ${ }^{(20)}$, in which patients with gallbladder carcinoma were similarly misdiagnosed at ultrasound as having cholecystitis with cholelithiasis. Gohil et al. ${ }^{(21)}$ had earlier alluded to this possibility, noting that cholelithiasis and cholecystitis were close differentials of gallbladder carcinoma.

The sensitivity, specificity, and accuracy of ultrasound in diagnosing choledocholithiasis were $70.7 \%, 93.7 \%$ and $88.8 \%$, respectively. We obtained a higher level of accuracy than Uphadyaya et al. ${ }^{(17)}$, who recorded the value of $63.1 \%$. Improved resolution of the equipment used in the present study and increased diagnostic efforts to determine the cause of jaundice may account for this difference. In this study, 3 patients presenting with choledocholithiasis had a normal biliary tree - probably because the condition was still early, hence ductal obstruction had not yet occurred. These cases were misdiagnosed as non-obstructive jaundice, similarly to the experiences of Upadhyaya et al. ${ }^{(17)}$

The sensitivity of ultrasound in diagnosing cholangiocarcinoma in our study was $100 \%$, which is at variance with the study by Robledo et al.(22) reporting that the detection of bile duct cancer varies from $21 \%$ to $90 \%$, with the distal common bile duct having the lowest percentage. Both proximal and distal bile duct cancers were encountered in this study, and the sensitivity and accuracy of ultrasonography were not impaired despite this. The higher sensitivity we encountered may be attributable to the fact that all extra-hepatic bile duct masses seen in this study were polypoidal intra-luminal tumors. This was also the finding of Anand et al. (23), who reported an accuracy of $100 \%$ for polypoidal intra-luminal tumors, $13 \%$ for sclerosing tumors, and $29 \%$ for exophytic tumors.

The non-dilatation of the biliary ducts in 5 of our patients probably accounted for the misdiagnosis of 2 cases of cholangitis and 3 cases of choledocholithiasis on ultrasound. They were mistakenly diagnosed as cholecystitis and cholelithiasis, respectively. Sample et al. ${ }^{(24)}$ had a similar experience, reporting that $10 \%$ of their patients had nondilated biliary trees due to sclerosing cholangitis which they misdiagnosed as non-obstructive jaundice. These cases emphasize the point that in the absence of ductal dilatation on ultrasonography, the presence of a clinical history of obstructive jaundice with biochemical findings also indicating an obstructive etiology should prompt further diagnostic investigations using other imaging modalities ${ }^{(25)}$.

\section{Conclusion}

In conclusion, ultrasonography is a helpful tool for evaluating patients with features of surgical jaundice in resource-poor 
settings. It can demonstrate both benign and malignant causes of obstructive jaundice. The most common causes of obstructive jaundice in our study were choledocholithiasis and carcinoma of the head of the pancreas for benign and malignant causes, respectively. The level of sensitivity obtained in the study is quite comparable to that reported in some previous studies for CT, MRCP, PTC, and $\operatorname{ERCP}^{(3,15,26,27)}$. We believe that the meticulous, protocol-driven ${ }^{(11,12)}$ use of ultrasound for the diagnosis of obstructive jaundice would further help to improve surgical management.

\section{References}

1. Chalya PL, Kanumba ES, McHembe M: Etiological spectrum and treatment outcome of obstructive jaundice at a University teaching Hospital in northwestern Tanzania: a diagnostic and therapeutic challenges. BMC Res Notes 2011; 4: 147.

2. Singh A, Mann HS, Thukral CL, Singh NR: Diagnostic accuracy of MRCP as compared to ultrasound/CT in patients with obstructive jaundice. J Clin Diagn Res 2014; 8: 103-107.

3. Dodiyi-Manuel A, Jebbin N: Management of obstructive jaundice: experience in a tertiary centre in Nigeria. Asian J Med Clin Sci 2013; 2: 21-23.

4. Gameraddin M, Omer S, Salih S, Elsayed SA, Alshaikh A: Sonographic evaluation of obstructive jaundice. Open J Med Imaging 2015; 5: 24-29.

5. Bhargava S, Thingujam U, Bhatt S, Kumari R, Bhargava S: Imaging in obstructive jaundice: a review with our experience. JIMSA 2013; 26: 43-46.

6. Tse F, Barkun JS, Romagnuolo J, Friedman G, Bornstein JD, Barkun AN: Nonoperative imaging techniques in suspected biliary tract obstruction. HPB 2006; 8: 409-425.

7. Idowu BM, Onigbinde SO, Ebie IU, Adeyemi MT: Gallbladder diseases in pregnancy: sonographic findings in an indigenous African population. J Ultrason 2019; 19: 269-275.

8. Madhusudhan KS, Gamanagatti S, Srivastava DN, Gupta AK: Radiological interventions in malignant biliary obstruction. World J Radiol 2016; 8: 518-529.

9. Admassie D, H/Yesus A, Denke A: Validity of ultrasonography in diagnosing obstructive jaundice. East Afr Med J 2005; 82: 379-381.

10. Karki S, Joshi KS, Regmi S, Gurung RB, Malla B: Role of ultrasound as compared with ERCP in patient with obstructive jaundice. Kathmandu Univ Med J 2013; 11: 237-240.

11. Kreimer F, Cunha DJ, Ferreira CC, Rodrigues TM, Fulco LG, Godoy ES. Comparative analysis of preoperative ultrasonography reports with intraoperative surgical findings in cholelithiasis. Arq Bras Cir Dig 2016; 29: 26-29.

12. Dlamini N, Goodier M: Adequacy of ultrasound reports in patients presenting with obstructive jaundice at a tertiary hospital radiology department. South Afr J Radiol 2016; 20: 1-8.

13. Middleton WD, Kurtz AB, Hertzberg BS, Thrall JH: Ultrasound. Mosby, St. Louis 2004.

14. Rahman GA, Yusuf IF, Faniyi AO, Etonyeaku AC: Management of patients with obstructive jaundice: experience in a developing country. Niger Q J Hosp Med 2011; 21: 75-79.

\section{Conflict of interest}

Authors do not report any financial or personal connections with other persons or organizations which might negatively affect the contents of this publication and/or claim authorship rights to this publication.

15. Kumar M, Prashad R, Kumar A, Sharma R, Acharya SK, Chattopadhyay TK: Relative merits of ultrasonography, computed tomography and cholangiography in patients of surgical obstructive jaundice. Hepatogastroenterology 1998; 45: 2027-2032.

16. Dewbury KC, Joseph AE, Hayes S, Murray C: Ultrasound in the evaluation and diagnosis of jaundice. Br J Radiol 1979; 52: 276-280.

17. Upadhyaya V, Upadhyaya DN, Ansari MA, Shukla VK: Comparative assessment of imaging modalities in biliary obstruction. Indian J Radiol Imaging 2006; 16: 577-582.

18. Ghimire R, Lohani B, Pradhan S: Accuracy of ultrasonography in evaluation of level and cause of biliary obstruction: a prospective study. Kathmandu Univ Med J 2005; 3: 17-21.

19. D’Onofrio M, Zamboni G, Tognolini A, Malago R, Faccioli N, Frulloni L et al.: Mass-forming pancreatitis: value of contrast-enhanced ultrasonography. World J Gastroenterol 2006; 12: 4181-4184.

20. Alatise OI, Ndububa DA, Ojo OS, Agbakwuru EA, Adekanle O, Arowolo OA: Pancreatic cancer in Nigeria: Past, present and future. Niger J Gastroenterol Hepatol 2009; 1: 61-73.

21. Gohil YM, Patel SB, Goswami KG, Shah S, Soni H: Ultrasonography in obstructive jaundice - a pictorial essay. Indian J Radiol Imaging 2006; 16: 477-481.

22. Robledo R, Muro A, Prieto ML: Extrahepatic bile duct carcinoma: US characteristics and accuracy in demonstration of tumors. Radiology 1996; 198: 869-873.

23. Anand M, Nicholson D, Coombs B, Silverman P, Karani K, Amin Z: Cholangiocarcinoma imaging. Medscape Radiol 2019 (cited 2020 Feb 15).Available from: https://emedicine.medscape.com/article/365065overview\#showall

24. Sample WF, Sartl DA, Goldstein LI, Weiner M, Kadell BM: Gray-scale ultrasonography of the jaundiced patient. Radiology 1978; 128: 719725 .

25. Deitch EA: The reliability and clinical limitations of sonographic scanning of the biliary ducts. Ann Surg 1981; 194: 167-170.

26. Feng GH, Cai Y, Jia Z, Yang DQ, Chen H, Jin HC et al.: Interventional therapy of malignant obstructive jaundice. Hepatobiliary Pancreat Dis Int 2003; 2: 300-302.

27. Gelan EA, Abdlhadi M, Bekele M, Tsehay A, Lemmu B: A retrospective analysis of etiological spectrum, clinical presentation, treatment and outcome of extra hepatic biliary tree obstruction at a tertiary teaching hospital in Addis Ababa, Ethiopia. Ethiop Med J 2019; 57: 125-132. 